\title{
Emotional structure of jokes: A corpus-based investigation
}

\author{
Yu-Chen Chan* \\ Institute of Learning Sciences, National Tsing Hua University, Hsinchu 30013, Taiwan
}

\begin{abstract}
Although considerable attention has been paid to the cognitive structure of humor, its emotional structure tends to be overlooked. Humor is often associated with the single emotion of mirth or amusement, while other aspects of its rich emotional structure are ignored. The purpose of the present study was to explore this structure by analyzing the content of a Taiwanese corpus of 204 'negative' jokes to identify the basic emotion was induced and the emotional shift pattern of the joke. Additionally, the corpus might be used to compare emotional reversal jokes (negative to positive emotion) and regular jokes (neutral to positive emotion) as an aid when preparing materials for use in functional Magnetic Resonance Imaging (fMRI) investigations on the neural substrates of humor. In terms of basic emotions, 82 fear jokes, 61 disgust jokes, 42 sadness jokes and 19 anger jokes were found. The most common type of emotional shift was from negative to positive, with the punch lines of 114 jokes providing relief from the negative emotion by either diverting attention away from it or dissolving it entirely.
\end{abstract}

Keywords: Joke corpus, emotional reversal, emotional shift pattern, negative joke, humor

\section{Introduction}

A joke consists of two processes, namely cognition and emotion. The comprehension of a joke is derived from cognitive processes of incongruity and resolution: when it reaches the stage of resolution, feelings of amusement are elicited from appreciating the joke. Considerable efforts have been made in behavioral studies on theorizing the cognitive processes that underlie humor [1-4]. Recently, Chan et al. further investigated more details of the process of comprehending and appreciating humor by examining distinctive regions of the brain in functional neuroimaging studies [5,6]. Most of these theories are variations of Suls' incongruity-resolution mechanism. Suls' theory can be illustrated by considering the traditional setup and punch line structure of a joke [3]. The setup provides necessary details to follow the joke, establishing a context which enables expectations to be formed of what is likely to follow. The punch line then generates humor through an unexpected 'twist' which is not congruent with the information in the setup.

When the listener or reader resolves this incongruity in a playful manner, mirth or amusement is generated and this, of course, is why humor is associated with a positive emotional state. In other words, previous studies mainly focused on regular jokes (neutral to positive emotion). However, not

\footnotetext{
${ }^{*}$ Corresponding author: Yu-Chen Chan, No. 101, Sec. 2, Kuang-Fu Rd., Hsinchu, Taiwan. Tel.: +886-3-5715131 ext. 34554; Fax: +886-3-573-9254; Email: ycchan@mx.nthu.edu.tw.
}

0959-2989/14/\$27.50 @ 2014 - IOS Press and the authors. 
all jokes have a happy ending; in fact, do jokes with different emotional shift patterns lead to the reversal of diverse emotions?

Humor as play is proposed by Michael Apter [7]. Therefore, what is play? Play is a state of mind, a special mental attitude toward the world with one's actions in it. A playful frame of mind is a paratelic state (present-directed), different from telic state (goal-directed) that underlies more serious activities. According to the "reversal theory", the listener transfers back and forth between these two states of mind at different time throughout a typical day. Humor that involves topics such as parodies of horror movies, sick jokes, etc. would normally arouse feelings of horror, revulsion, or disgust. In a telic state, high arousal is unpleasant (anxiety) and low arousal is preferred (relaxation), whereas in a paratelic state, low arousal is unpleasant (boredom) and high arousal is enjoyable (excitement) [8].

\subsection{Negative emotions: fear, disgust, sadness and anger}

Jokes designed to elicit the four negative emotions on Ekman's original list of six basic emotions (happiness, fear, anger, disgust, sadness, surprise) [9] are the focus of this section. Ekman later expanded this list to 15 emotions [10] by adding embarrassment, guilt, contempt and shame. However, embarrassment, guilt, contempt and shame are more subtle and complex, while anger, disgust, fear and sadness are triggered by a wider variety of stimuli. Therefore, the latter emotions appear to be more suitable for this initial exploration in terms of collecting and analyzing jokes.

\subsection{Eliciting negative emotions: joke structure and content}

Cognitive theories of joke processing typically posit that both the setup and punch line introduce a cognitive frame or script. When juxtaposed, an incongruity emerges between the frame introduced in the punch line and the expectations engendered by the setup. Cognitive frames may also induce particular emotional experiences, resulting in different emotional structures or shift patterns, as illustrated by Figure 1 .

The present focus is on negative emotions, and since only jokes with a negative component are included in the corpus, the four shift patterns that do not include a negative component (neutral-toneutral, neutral-to-positive, positive-to-neutral and positive-to-positive) are not considered here. Therefore, in the setup, only negative and neutral jokes are listed in Figure 1.

Setup

(Cognitive Frame 1)

NEGATIVE

NEUTRAL

NEGATIVE
Punch Line

(Cognitive Frame 2)

$(\mathrm{MIRTH}+)$ POSITIVE

$($ MIRTH +) NEGATIVE

$($ MIRTH +) NEGATIVE

\section{Emotional Shift Pattern}

NEG-POS

NEU-NEG

NEG-NEG

Fig. 1. Emotional shift patterns resulting from different combinations of emotions elicited by joke setups and punch lines. 
Since the second stage always involves mirth at least as shown in Figure 1 and mirth on its own is assumed to be a positive emotion, 'NEGATIVE TO MIRTH + POSITIVE' and 'NEGATIVE TO MIRTH + NEUTRAL' jokes are combined in the category 'NEG-POS.' 'Mirth' is parenthesized for jokes ending with a negative emotion to highlight the expectation that the positive emotion derived from 'getting' the joke is likely to be diminished, altered or even eliminated entirely, depending on the nature and intensity of the accompanying negative emotion.

The resulted three shift patterns suggest distinct possibilities in terms of the emotional effect induced by the joke. Successful instances of the first category, 'NEG-POS', should achieve a sense of relief as the initial negative emotion gives way to a feeling of amusement. Jokes of the second type, NEU-NEG, are likely to introduce an 'unpleasant surprise.' Finally, on the other hand, jokes in the NEG-NEG category should achieve their effects by intensifying the negative emotion that appeared in the setup. The three shift patterns are shown in Figure 1.

The present study seeks to take a step in this direction by analyzing the stimuli in negative jokes. The implications of the present findings for an emotional reversal in negative jokes are open to future investigation and further revelations in neuroimaging studies.

A joke is part of the emotion expression, which is an important topic in biomedical research in human science and clinic medicine. In terms of the structure of a joke and the content of the present study, a content analysis was performed on a Taiwanese corpus of negative jokes in order to explore the shift patterns and content of jokes that elicited negative emotions of fear, disgust, sadness and anger. Additionally, the corpus might be used to compare emotional reversal jokes (negative to positive emotion, NEG-POS) and regular jokes (neutral to positive emotion, NEU-POS) from Chan et al.'s studies $[5,6]$ as an aid in preparing material for use in fMRI investigations on the neural substrates of humor.

\section{Methods}

\subsection{Material}

In the initial stage of research, a corpus of jokes written in Chinese (traditional characters) and containing elements of fear, disgust, sadness, or anger were extensively collected from the Internet, books, magazines and previous studies of joke-rating norms [11]. Jokes involving sexual or political content were excluded and those with similar plots were removed, leaving 204 jokes with negative emotional content. One reason for excluding such jokes was that there appeared to be gender differences in response to them [11].

\subsection{Coding categories}

In terms of joke classification, the four kinds of negative emotions discussed above, namely fear, disgust, sadness and anger, were used to categorize the jokes. Jokes eliciting fear are sometimes called 'fear jokes' in English, while jokes eliciting disgust called 'sick jokes' or 'gross jokes.' Here the four types of joke are simply referred to as 'fear jokes,' 'disgust jokes,' 'sadness jokes' and 'anger jokes', respectively. The three shift patterns are negative to positive (NEG-POS), neutral to negative (NEUNEG) and negative to (more) negative (NEG-NEG).

The resulted set of codes includes 12 distinct categories (four types of emotion $\times$ three types of emotional shift), all of which are illustrated in Table 1. 
Table 1

Coding categories (emotion type $\times$ shift pattern)

\begin{tabular}{lll}
\hline Negative emotion type & Change of emotional valence & Emotional shift pattern \\
\hline \multirow{3}{*}{ Fear } & Negative (fear) to positive (mirth/amusement) & NEG-POS \\
& Neutral (calm) to negative (fear) & NEU-NEG \\
& Negative (fear) to negative (intensified fear) & NEG-NEG \\
\hline \multirow{3}{*}{ Disgust } & Negative (disgust) to positive (mirth/amusement) & NEG-POS \\
& Neutral (calm) to negative (disgust) & NEU-NEG \\
& Negative (disgust) to negative (intensified disgust) & NEG-NEG \\
\hline \multirow{3}{*}{ Sadness } & Negative (sadness) to positive (mirth/amusement) & NEG-POS \\
& Neutral (calm) to negative (sadness) & NEU-NEG \\
& Negative (sadness) to negative (intensified sadness) & NEG-NEG \\
\multirow{3}{*}{ Anger } & Negative (anger) to positive (mirth/amusement) & NEG-POS \\
& Neutral (calm) to negative (anger) & NEU-NEG \\
& Negative (anger) to negative (intensified anger) & NEG-NEG
\end{tabular}

\subsection{Coding procedure}

Each joke was categorized by seven raters, one of which is the author of this paper and the other six are researchers on humor. The categories were firstly explained to the raters before showing them one sample joke for each category and the researcher explained why the joke was chosen to exemplify that particular category. The raters were given time to read each sample joke and were asked if they had any comments or questions about the categories assigned. After answering the questions, the raters were asked to analyze all of the jokes independently via a computer. The jokes were presented in seven random blocks with approximately the same size and the raters were instructed to view each block in a separate sitting over a three-day period in order to avoid "fun accumulation and fun fatigue effects" [12].

They were asked to firstly categorize the setup and punch line of each joke according to the type of negative emotion jokes were designed to induce (fear, disgust, sadness or anger), and according to their emotional shift pattern (NEG-POS, NEU-NEG or NEG-NEG). Thus, each joke was assigned to 1 of 12 possible categories (four types of emotion $\times$ three emotional shift patterns). A disgust joke is shown below as a sample.

\subsubsection{Sample joke: NEG-POS (disgust)}

Setup: A group of medical students gather around a surgical table with a corpse on it, listening to their professor, "To be a good doctor, you must possess two critical qualities: First, you must not be disgusted by anything involved in the human body." With that, he inserts his finger into the anus of the body, pulls it out and sticks his finger in his mouth. Then he tells his students, "Now, I want all of you to do the same." The students hesitate, but eventually they all do it.

Punch line: Then the professor says, "The next quality is the power of observation. Had you been more observant, you would have noticed that I inserted my middle finger but licked my index finger."

\subsection{Inter-rater reliability}

Interrater reliability was evaluated using an online Cohen's calculator [13]. Brennan and Prediger suggest using free-marginal kappa when raters are not forced to assign a pre-specified number of cases 
to each category. The coefficient kappa for the percentage of overall agreement between multiple raters was 0.88 and the free-marginal multi-rater kappa was 0.87. The rule of thumb is that a kappa of 0.70 or above indicates adequate interrater agreement [14]. Hence, it is concluded that the level of agreement found in the present study was satisfactory. When there was disagreement, each rater read and categorized each joke and the seven raters discussed the jokes together to achieve a consensus.

\section{Results}

\subsection{Joke type and emotional shift patterns of negative jokes}

The resulted distribution of the 204 jokes into 12 categories is shown in Table 2 . In terms of basic emotions, 82 fear jokes were the most common emotional type, followed by 61 disgust jokes, 42 sadness jokes and 19 anger jokes successively. As for the three emotional shift patterns, most jokes followed the NEG-POS ('relief') pattern (55.9\%), with NEG-NEG ('intensification') jokes being the second largest category (29.4\%) and NEU-NEG ('unpleasant surprise') jokes being the least frequent $(14.7 \%)$.

\subsection{Distribution by joke type and emotional shift pattern}

More interesting are the emotional shift patterns for different types of emotion. A Pearson's Chisquare analysis showed a significant relationship between the type of emotion and type of change $\left(\chi^{2}(6)=93.46, p<.001\right)$, indicating that there is a relationship between the emotion type and shift pat-

Table 2

Negative Jokes, by Joke and Shift Types

\begin{tabular}{|c|c|c|c|c|c|c|}
\hline \multirow[b]{2}{*}{ Shift Type } & & \multicolumn{5}{|c|}{ Joke Type } \\
\hline & & $\begin{array}{l}\text { Fear } \\
\text { Jokes }\end{array}$ & $\begin{array}{l}\text { Disgust } \\
\text { Jokes }\end{array}$ & $\begin{array}{l}\text { Sadness } \\
\text { Jokes }\end{array}$ & $\begin{array}{l}\text { Anger } \\
\text { Jokes }\end{array}$ & Total \\
\hline \multirow{3}{*}{ NEG-POS } & Number & 64 & 6 & 32 & 12 & 114 \\
\hline & By shift type & $78.0 \%$ & $9.8 \%$ & $76.2 \%$ & $63.2 \%$ & $55.9 \%$ \\
\hline & ASR & $5.2^{*}$ & $-8.7 *$ & $3.0^{*}$ & .7 & \\
\hline \multirow{3}{*}{ NEU-NEG } & Number & 8 & 12 & 4 & 6 & 30 \\
\hline & By shift type & $9.8 \%$ & $19.7 \%$ & $9.5 \%$ & $31.6 \%$ & $14.7 \%$ \\
\hline & ASR & -1.6 & 1.3 & -1.1 & $2.2 *$ & \\
\hline \multirow{6}{*}{ NEG-NEG } & Number & 10 & 43 & 6 & 1 & 60 \\
\hline & By shift type & $12.2 \%$ & $70.5 \%$ & $14.3 \%$ & $5.3 \%$ & $29.4 \%$ \\
\hline & ASR & $-4.4^{*}$ & $8.4^{*}$ & $-2.4^{*}$ & $-2.4^{*}$ & \\
\hline & Total & 82 & 61 & 42 & 19 & 204 \\
\hline & By shift type & $100.0 \%$ & $100.0 \%$ & $100.0 \%$ & $100.0 \%$ & $100.0 \%$ \\
\hline & By joke type & $40.2 \%$ & $29.9 \%$ & $20.6 \%$ & $9.3 \%$ & $100.0 \%$ \\
\hline
\end{tabular}

Note: *Adjusted standardized residuals (ASRs) are distributed according to the standard normal distribution with mean of 0 and standard deviation of 1 . ASRs with an absolute value greater than 2 were deemed significant while values less than 2 were insignificant.

tern. Using the contingency coefficient, the strength of association was $C=.56, p<.001$, indicating a moderate association between joke type and shift pattern. 
In NEG-POS jokes, the setup creates a negative emotional state but the 'surprise' in the punch line either dissipates this mood or distracts attention away from it, leaving a positive emotion of mirth or amusement generated by the humor itself. Interestingly, while this emotional shift type accounted for the majority of fear jokes (78\%), sadness jokes (76.2\%) and anger jokes (63.2\%), it characterized only $9.8 \%$ of disgust jokes.

In the NEU-NEG jokes, the setup does not create a particular mood or create a mildly positive mood, but the surprise found in the punch line is an unpleasant one, leaving the listener with a potential mix of amusement and negativity. Only a minority of the 204 jokes fell into this category: $9.8 \%$ of fear jokes and $9.5 \%$ of sadness jokes, but rising to $19.7 \%$ of disgust jokes and $31.6 \%$ of anger jokes.

In terms of jokes in the NEG-NEG category, the setup creates a negative feeling, and the surprise twist in the punch line achieves its effect by intensifying that negative feeling. Only 5.3\% of anger jokes were in the intensification category, compared to similar proportions of $12.2 \%$ and $14.3 \%$ for fear and sadness jokes respectively and a striking percentage of $70.5 \%$ for disgust jokes.

The comparison between the shift type and each joke type may provide further illumination. The preferred structure of fear and sadness jokes clearly seems to be NEG-POS ( $78 \%$ and $76.2 \%$, respectively). This suggests that the intended experience is a relief from these emotions. Most anger jokes also follow this shift pattern, again suggesting a preference for a relief from feelings of anger, indignation or frustration. However, for anger, more of the remaining jokes were in the NEU-NEG category, with only one joke characterized by the NEG-NEG pattern. Jokes in the NEU-NEG category may mirror a social experience of witnessing the exposure of unethical, dishonest or immoral behavior. The only anger joke displaying the NEG-NEG shift pattern also appeared to reflect common social experiences.

Finally, the disgust jokes reveal a very different distribution, since most of them involve the intensification of the feeling of disgust, and the second largest category is NEU-NEG, implying a 'disgust surprise'. Less than $10 \%$ of these jokes follow the NEG-POS pattern, clearly suggesting that, for those who like to tell these jokes, the point of the experience is to make listeners feel disgusted.

\section{Conclusion}

This study has attempted to redress the relative lack of attention in the cognitive structure of humor compared with its emotional structure. In other words, it seeks to categorize the internal emotional structure of negative jokes in terms of the initial emotion jokes are designed to induce, the final emotion, and the emotional 'shift'. The study also provides a description of the emotionally-relevant stimuli attributes of these negative jokes.

A corpus of 204 jokes which appeared to have been designed to elicit negative emotions were analyzed in two stages. First, jokes within each category of four types of emotion (fear, disgust, sadness, anger) were categorized according to the nature of the emotional shift jokes were designed to induce. Most jokes were categorized as 'NEG-POS', indicating that a negative emotional context was established in the cognitive frame introduced by the setup, and then shifted to a positive emotion in the punch line by either dissolving or distracting attention away from the initial elicitors of negative feelings. The second most common category was 'NEG-NEG', which refers to jokes that established a negative emotional context in the setup and then intensified this feeling in the punch line. The least used category was 'NEU-NEG', which contained jokes that established a neutral or mildly positive emotional context in the setup, but then introduced an 'unpleasant surprise' in the punch line, eliciting a negative emotional state. 
Interestingly, different negative emotions had different patterns. Fear and sadness jokes were distributed almost identically across the different categories: most of them were 'NEG-POS', meaning that the punch line brought a sense of relief from negative feelings, with just over 10 percent following the 'NEG-NEG' pattern and nearly 10 percent following the 'NEU-NEG' pattern.

The findings of the present study have implications for the understanding of humorous discourse and its role in emotional regulation, within the context of which it is difficult, but important, to move from a negative emotional state to a positive one. In this context, jokes (and humor more generally) are of obvious importance, since they possess a kind of 'instant reversal mechanism', which is capable of changing both the cognitive and affective appraisal of a situation. One type of emotional regulation involves the cognitive re-appraisal of a situation [15], and the structure of a joke involves just such a re-appraisal, with the punch line triggering a re-interpretation of the scenario presented in the setup.

The results of the present study demonstrate that jokes have their own complex emotional structure, which involves particular types of emotional shifts and reversals. Furthermore, they provide evidence that jokes do elicit the predicted types of emotions and do result in the predicted shift or change in emotional states, from negative to positive. Fear jokes are distributed almost identically across different categories, mostly 'NEG-POS'. Future studies could focus on an advanced investigation by comparing the NEG-POS pattern of emotional reversal jokes and the NEU-POS pattern of regular jokes, thereby distinguishing neural substrates and verifying the neural circuit path mode of the joke.

\section{Acknowledgement}

The author wishes to thank Joseph P. Lavallee and Hsueh-Chih Chen for their help. This research was partially supported by the "Identifying the Neural Substrates of the Ambiguity-Humor Interaction: An fMRI study" of the National Tsing Hua University (NTHU), sponsored by the Ministry of Science and Technology under Grant No. NSC 102-2410-H-007-072. This research is also partially supported by the "Aim for the Top University Project" of National Taiwan Normal University (NTNU), sponsored by the Ministry of Education, Taiwan, R.O.C.

\section{References}

[1] M.J. Apter, The Experience of Motivation: The Theory of Psychological Reversals, Academic Press, San Diego, 1982.

[2] S. Attardo, Linguistic Theories of Humor, Hawthorne, ed., Mouton de Gruyter, New York, 1994.

[3] J.M. Suls, Two-stage model for the appreciation of jokes and cartoons: Information-processing analysis, in: The Psychology of Humor, J.H. Goldstein and P.E. McGhee, eds., Academic Press, San Diego, CA, 1972, pp. 81-100.

[4] R.S. Wyer and J.E. Collins, A theory of humor elicitation, Psychological Review 99 (199), 663-688.

[5] Y.C. Chan, T.L. Chou, H.C. Chen and K.C. Liang, Segregating humor comprehension and elaboration process of verbal jokes: An fMRI study, NeuroImage 61 (2012), 899-906.

[6] Y.C. Chan, T.L. Chou, H.C. Chen, Y.C. Yeh, J.P. Lavalle, K.C. Liang and K.E. Chang, Towards a neural circuit model of verbal humor processing: An fMRI study of the neural substrates of incongruity detection and resolution, NeuroImage 66 (2013), 169-176.

[7] M.J. Apter, Motivational styles in everyday life: A guide to reversal theory, American Psychological Association, Washington DC, 2001.

[8] M.J. Apter, A structural-phenomenology of play, in: Adult Play: A Reversal Theory Approach, J.H. Kerr and M.J. Apter, eds., Swets \& Zeitlinger, Amsterdam, 1991, pp. 13-29.

[9] P. Ekman, Expression and the nature of emotion, in: Approaches to Emotion, K. Scherer and P. Ekman, eds., Erlbaum, Hillsdale, NJ, 1984, pp. 319-344. 
[10] P. Ekman, Basic emotions, in: Handbook of Cognition and Emotion, T. Dalgleish and M. Power, eds., John Wiley and Sons Ltd, Sussex, 1999, pp. 45-60.

[11] C.M. Cheng, H.C. Chen, Y.C. Chan, Y.C. Su and C.C. Tseng, Taiwan corpora of Chinese emotions and relevant psychophysiological data-normative data for Chinese jokes, Chinese Journal of Psychology 55 (2013), 555-569.

[12] L.J. Martin, Psychology of aesthetics: Experimental prospecting in the field of the comic, American Journal of Psychology 16 (1905), 35-118.

[13] J.J. Randolph, Online kappa calculator, 2008, Available at: http://justus.randolph.name/kappa.

[14] R.L. Brennan and D.J. Prediger, Coefficient kappa: Some uses, misuses, and alternatives, Educational and Psychological Measurement 41 (1981), 687-699.

[15] J.J. Gross, Emotion regulation, in: Handbook of Emotions $\left(3^{\text {rd }}\right)$, M. Lewis, J.M. Haviland-Jones and L.F. Barrett, eds., Guildford, New York and London, 2008, pp. 497-512. 